\title{
Preparation and Characterization of Nanofibrillated Cellulose from Waste Sugarcane Bagasse by Mechanical Force
}

\author{
Kehong Zhang, ${ }^{*, a, b}$ Yuhang Su, ${ }^{b}$ and Hui Xiao ${ }^{a}$ \\ The effect of different mechanical force application methods was \\ investigated relative to the structure and properties of nanofibrillated \\ cellulose (NFC) from sugarcane bagasse. The NFC was prepared by \\ grinding, high pressure homogenization, and ultrasonication with chemical \\ pretreatment. Fiber morphology, crystalline, chemical structures, and \\ tensile property were analyzed to reveal the mechanisms of NFC \\ production behind different mechanical treatments. The results showed \\ that compared with grinding treatment, high pressure homogenization and \\ ultrasonic treatment can obviously increase the aspect ratio and size \\ uniformity of NFC. Ultrasonic treatment gave the best results. The \\ combination of grinding, high pressure homogenization, and ultrasonic \\ treatment resulted in NFC with the average diameter of $23.18 \mathrm{~nm}$. With \\ the shearing action of grinding, high pressure homogenization, and \\ ultrasonic treatment, the mechanical properties, crystallinity and thermal \\ stability of NFC were gradually enhanced. This results demonstrated that \\ a very low-value agricultural waste product can be easily converted to a \\ high performance nanocomposite with tensile strength of $153.6 \mathrm{MPa}$ and \\ strain at break of $8.83 \%$.
}

Keywords: Cellulose nanofibers; Sugarcane bagasse; Grinding; High pressure homogenization; Ultrasonic

Contact information: a: School of Light Textile Engineering and Art, Anhui Agricultural University, China, 230036; b: Fujian Universities and Colleges Engineering Research Center of Soft Plastic Packaging Technology for Food, China, 350300; *Corresponding author: zkh@ahau.edu.cn

\section{INTRODUCTION}

With the emphasis on environment protection, the harm of petroleum-based materials to the environment has received increasing attention. This consideration has prompted much research into the development of bio-based polymers to replace nonbiodegradable materials (Tang et al. 2012). As the most abundant biopolymer, cellulose has received increasing attention due to its wide availability, excellent performance, biocompatibility, low density, thermal stability, and environmental benefits. It is a homopolysaccharide, composed of $(\beta-1,4)$-linked-glucopyranose units (Abraham et al. 2011) with polymer chains connected by hydrogen bonds and van der Waals forces conferring strength and stability to the plant cell walls. In addition to its widespread use in fiber, paper, films, and polymers, the utilization of natural biomass for processing of novel material has recently attracted increasing interest (Mazalevska et al. 2011). Due to superior functionalities, such as extremely large, active surface area, and low cost, the production of NFC from renewable cellulose has gained attention. 
NFC consists of particles with nano-sized dimension generated from cellulose macromolecules comprising of both ordered crystallites domains and disordered amorphous domains. The advantages of NFC are its inherent biodegradability, renewability, high tensile strength $(10 \mathrm{GPa})$ and Young's modulus (100 to $160 \mathrm{GPa})$, large specific surface area, high aspect ratio, and unique morphology (Afra et al. 2013; Chen et al. 2013). There is a wide variety of wood and non-wood resources suitable for producing cellulose nanostructures.

The development of non-wood sources of cellulose is important because of the shortage of wood resources (Alila et al. 2013). By contrast, a serious challenge associated with agricultural intensification is the millions of tons of agricultural wastes generated each year. Approximately 180 million tons of bagasse is produced each year in the world (de Moraes Rocha et al. 2015). As an abundant lignocellulosic agro-industrial by-product obtained during sugar manufacturing, sugarcane bagasse fibers represent approximately $30 \%$ of the total weight, which are composed of approximately 40 to $50 \%$ semicrystalline cellulose, 25 to $35 \%$ amorphous hemicellulose, and 10 to $14 \%$ lignin (Ju et al. 2011). With the rapid progress in material science and engineering in recent year, many new processes and technologies have been developed to produce high-performance new materials using sugarcane bagasse as raw materials (Rossignolo et al. 2018; Khonngam and Salakkam 2019); particularly, in the production of pulp and paper and fermentation-based products (Bautista et al. 2019). Other products are obtained from processes related to bagasse including chemicals and metabolites such as alcohol and alkaloids, fungi, and animal food enriched with proteins and enzymes (Gil-López et al. 2019). Recently, the isolation of nanocellulose from sugarcane bagasse has been the focus of studies (Liu et al. 2020; Macías-Almazán et al. 2020). The mechanical performance of NFC can be tuned via modulation of their geometric shapes and stacking patterns in the matrix (Sun et al. 2018).

Mechanical defibrillation (mainly including ultrafine grinding, homogenization, microfluidization, and cryocrushing) is considered to be an essential step for NFC production, during which NFC particles are isolated by delamination of fiber cell wall under successive applications of mechanical force (Jiang et al. 2019). The use of conventional methods to extract biopolymers from agricultural wastes at high temperatures may lead to degradation of biopolymers and worsen their performance, which forces industry and researchers to replace traditional methods with alternative technologies. With simplicity, high efficiency and the lack of a requirement for organic solvents, high pressure homogenization (HPH) treatment is an efficient technology for isolation of cellulose fibers from biomass (Li et al. 2012; Wang et al. 2015; Ninomiya et al. 2018). The insolubility of natural cellulose in water and most organic solvents causes clogs in the valve of the homogenizer, and for this reason it is difficult to refine it uniformly. Therefore, a pretreatment of cellulose is essential prior to homogenization to avoiding clogging the homogenizer (Li et al. 2012).

In this study, NFC from sugarcane bagasse was successfully isolated by mechanical forces treatment generated from grinding, high pressure homogenization (HPH), and ultrasonication. The structural and physicochemical properties of NFC were studied by field emission scanning electron microscope (FE-SEM), X-ray diffraction (XRD), thermogravimetric analysis (TG), Fourier transformed infrared (FTIR) spectroscopy, and tensile property test. The main objective of the present study was to explore the morphological changes and mechanism of NFC under different mechanical treatments. 


\section{EXPERIMENTAL}

\section{Materials}

The sugarcane bagasse was obtained in the southern region of Anhui Province of China, during the harvest of September 2018. Hydrochloric acid $(\mathrm{HCl})$ was purchased from Aladdin Biochemical Technology Co., Ltd. (Shanghai, China). Acetic acid $\left(\mathrm{CH}_{3} \mathrm{COOH}\right)$, hexan $\left(\mathrm{C}_{6} \mathrm{H}_{14}\right)$, sodium chlorite $\left(\mathrm{NaClO}_{2}\right)$, and potassium hydroxide $(\mathrm{KOH})$ were supplied from Sinopharm Chemical Reagent Co., Ltd. (Shanghai, China).

\section{Preparation of Nanocellulose from Sugarcane Bagasse}

The bagasse sample was washed thoroughly in running water to remove all sugar residues from freshly collected sugarcane bagasse. The dry bagasse was manually shredded into small pieces ( 1 to $3 \mathrm{~cm}$ ). A hot air oven drying (at $60{ }^{\circ} \mathrm{C}$ ) was used to further dry the cut bagasse. The bagasse was pulverized using a mill and then sieved to gain a bagasse powder with particle size of 420 to $630 \mu \mathrm{m}$.

The dried and ground bagasse samples were first defatted using a Soxhlet apparatus with $\mathrm{C}_{6} \mathrm{H}_{14}$ for $6 \mathrm{~h}$. Cellulose fibers was extracted from sugarcane bagasse using a threestep process. The defatted bagasse powder in $0.7 \mathrm{wt} . \% \mathrm{NaClO}_{2}$ solution was boiled under reflux at $\mathrm{pH} 4$ (adjusted by $\mathrm{CH}_{3} \mathrm{COOH}$ ) for $3 \mathrm{~h}$ to remove lignin. Subsequently, the material was treated by $2 \mathrm{wt} \% \mathrm{KOH}$ solution for $2 \mathrm{~h}$ at $90{ }^{\circ} \mathrm{C}$ to remove most hemicelluloses. Finally, the whole mixture was treated $\mathrm{NaClO}_{2}$ at $\mathrm{pH} 4$ to 5 for $2 \mathrm{~h}, 5$ wt. $\%$ of $\mathrm{KOH}$ at $90{ }^{\circ} \mathrm{C}$ for $2 \mathrm{~h}$, and $1 \mathrm{wt} . \%$ of $\mathrm{HCl}$ solution at $85^{\circ} \mathrm{C}$ for $2 \mathrm{~h}$, to obtain purified cellulose. To obtain NFC from the bagasse cellulose, the method of mechanical force step by step refinement was employed. The $1 \mathrm{wt} \%$ NFC suspension was prepared by dispersing the purified cellulose into water. The purified cellulose suspension was poured into a grinder (IKN CMSD2000, Neustadt, Germany) with a rotational speed of $1500 \mathrm{rpm} / \mathrm{min}$ and a grindstone gap of -9 for three times to prepare grinding treated NFC suspension (GT). Next, the $1 \mathrm{wt} . \%$ NFC suspension prepared by grinding was treated five times in a high pressure homogenizer (SCIENTZ Scientz-150, Ningbo, China) under the condition of an average pressure of 1000 bar to obtain NFC prepared by the combination of grinding and high pressure homogenization treatment (GT-HPH). Finally, the $1 \mathrm{wt} \%$ GT-HPH treated NFC suspension was placed in the ultrasonic cell pulverizer (LAWSON DH-1200E, Ningbo, China) and treated by ultrasonic for $30 \mathrm{~min}$ at $900 \mathrm{~W}$ to obtain NFC prepared by the combination method of grinding, high pressure homogenization, and ultrasonic waves (GT-HPH-U).

\section{Preparation of NFC Films}

The NFC suspensions obtained by different mechanical forces treatment were filtered through a $0.22 \mu \mathrm{m}$ microporous filter membrane to obtain an NFC wet film. After removal from the vacuum filtration device, the two sides of the NFC wet film were covered with microporous filter membranes and coarse filter papers in turn. The sample was sandwiched between two glass plates with the pressure of $400 \mathrm{~N}$ and oven-dried at $80{ }^{\circ} \mathrm{C}$ for $24 \mathrm{~h}$ to obtain NFC.

\section{Characterisations and Measurements}

Morphological properties

The morphology of the NFC was analyzed with a scanning electron microscope (Hitachi S-4800, Tokyo, Japan) after being coated with platinum using sputtering technique 
to make them conductive. The SEM images were taken at the acceleration voltage of $3 \mathrm{kV}$ and under different magnifications.

The longitudinal surface of fibers was analyzed; the diameter of the NFC was measured from the SEM images using ImageJ software. The diameters of approximately 150 NFC was measured for each sample.

\section{$X$-ray diffraction analysis}

The X-ray diffractometer (Panalytical X 'Pert MPD, Almelo, Netherlands) equipped with an $\mathrm{X}$-ray generator $(\lambda=0.154 \mathrm{~nm})$ and with $\mathrm{Cu}-\mathrm{K} \alpha$ radiation $(K=1.54056$ $\mathrm{A}^{\circ}$ ) was operated over the $2 \theta$ range of 5 to $40^{\circ}$ with a scan step of $0.02^{\circ}$, at a scan rate of 5 $\%$ min.

The acceleration voltage and current were set to $40 \mathrm{kV}$ and $30 \mathrm{~mA}$, respectively. The degree of crystallinity was measured by empirical peak-height method (Segal et al. 1959), as represented in Eq. 1,

$$
C r=\frac{I_{200}-I_{a m}}{I_{200}} \times 100
$$

where $C_{\mathrm{r}}$ is the crystallinity, $I_{200}$ is the intensity value for the crystalline cellulose, and $I_{\mathrm{am}}$ is the intensity value for the amorphous cellulose. X-Ray diffraction data were analyzed using Origin 8.5 software (Northampton, MA, USA), based on the Gaussian function in order to do de-convolution and find individual peak regions.

\section{Thermogravimetric analysis}

Thermogravimetric analysis measurements (TG) were carried out with a Synchronous Thermal Analysis (Mettler TGA/DSC 1/1100, Zurich, Switzerland). The thermal stability of the samples was evaluated at heating rate of $10{ }^{\circ} \mathrm{C} / \mathrm{min}$, from 50 to $600{ }^{\circ} \mathrm{C}$. All measurements were performed under a nitrogen atmosphere with a gas flow of $20 \mathrm{~mL} / \mathrm{min}$ in order to prevent thermoxidative degradation.

\section{FIIR spectral analysis}

The FTIR spectra of original cellulose and NFC treated by different treatment methods were measured using a Bruker Optic Tensor 27 spectrometer (Karlsruhe, Germany). The samples were dried at $80{ }^{\circ} \mathrm{C}$ under vacuum for $12 \mathrm{~h}$ before analysis. The mixture of finely ground $\mathrm{NFC}$ and $\mathrm{KBr}$ powder at a mass ratio of 1:49 was compressed to form a disc.

All infrared spectra were recorded by an accumulation of 32 scans for each sample, with a resolution of $4 \mathrm{~cm}^{-1}$ at 4000 to $400 \mathrm{~cm}^{-1}$. The background spectrum in the absence of any sample was subtracted from the spectra of the individual samples.

\section{Mechanical properties}

Tensile tests of specimens were conducted on a universal testing machine (Labthink XLW-PC, Jinan, China) at room temperature. Gauge length was determined in the specimens at $20 \mathrm{~mm}$ and widths were $4 \mathrm{~mm}$. The rates for load cell and extension rate were $100 \mathrm{~N}$ and $1 \mathrm{~mm} / \mathrm{min}$, respectively. The tensile strength and modulus were determined from the obtained stress-strain curves. 


\section{RESULTS AND DISCUSSION}

\section{Morphological Characterization}
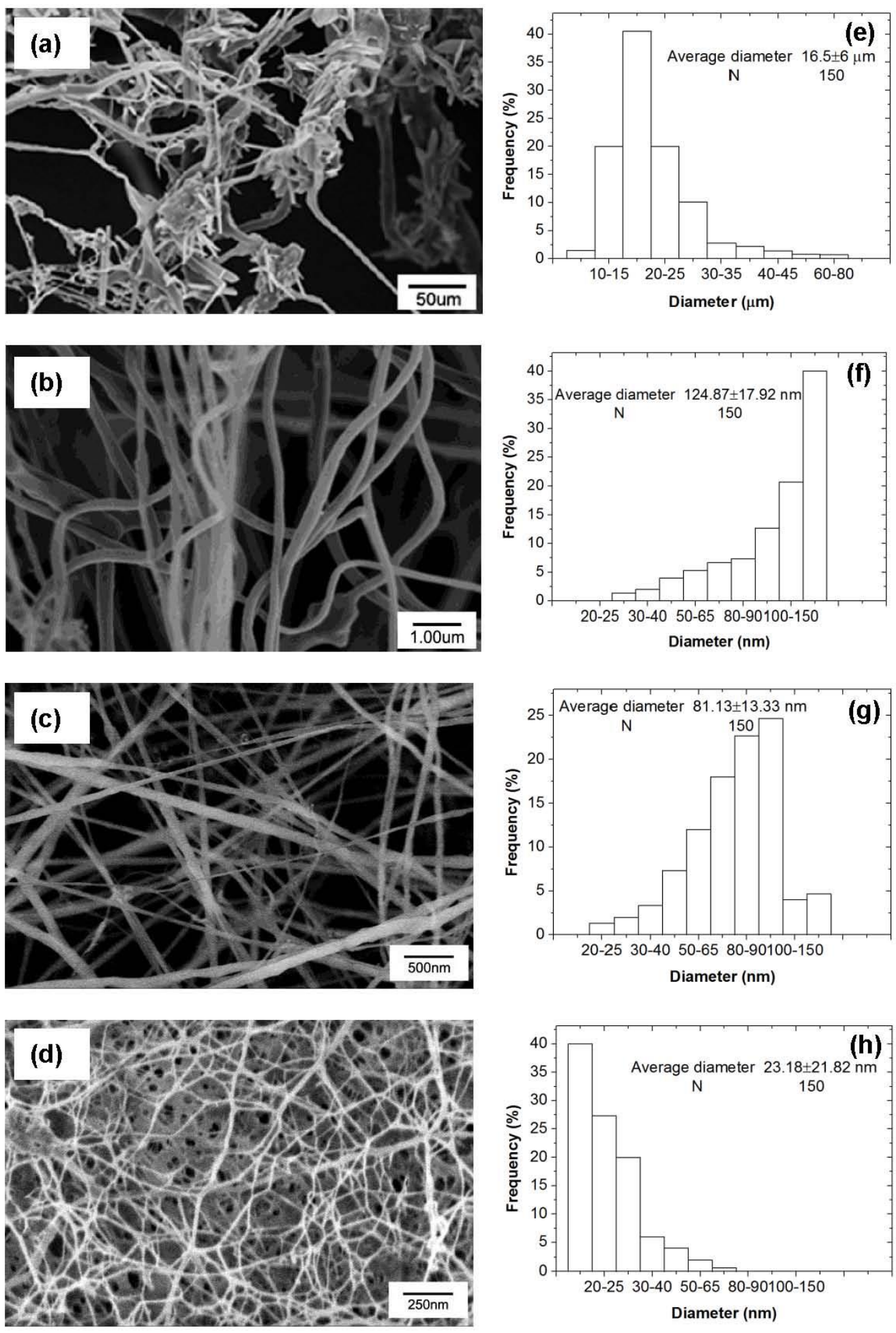

Fig. 1. FE-SEM micrographs of (a) chemical pretreated cellulose, (b) grinding treated NFC, (c) GT-HPH treated NFC, and (d) GT-HPH-U treated NFC. The diameter distribution and number of measured micro- and NFC are shown on Fig. 1 (e), (f), (g) and (h) accordingly. 
The structure and strengthening effect of NFC depend on their length, shape, and degree of nanocrystallization (Huq et al. 2012). Due to the extensive network via numerous intermolecular and intramolecular hydrogen bonds, the aggregated nanofibers should be pretreated by a mechanical force to obtain the homogeneous NFC without destroying in inherent structure (Liu et al. 2007). Selected NFC films were analyzed through SEM images to observe morphology and microstructural aspects (Fig. $1 \mathrm{a}-\mathrm{h}$ ). The NFC in film show a rod-like morphology (Fig. 1 a-d). The diameter distributions of cellulose and nanofiber produced by different mechanical treat method are shown in Fig. 1(e-h). The image in Fig. 1a shows that chemical pretreatment can remove the non-cellulosic impurities on the surface of cellulose and cause partial separation of the celluloses into the microsized fiber and a compact structure. Figures 1(b-d) show that the conversion from cellulose to NFC had been realized with the action of mechanical force. Comparing with Fig. $1 \mathrm{a}, \mathrm{b}$ and $\mathrm{c}$, it can be found that the degree of nanocrystallization of cellulose increased with the enhancement of mechanical force. With the treatment of HPH on NFC produced by grinding, the aggregated NFC was further converted to refined NFC with an average diameter of $81.13 \mathrm{~nm}$ under the high shearing action in the homogenizer. The diameter of most NFC ranged from 60 to $100 \mathrm{~nm}$. Under the treatment of ultrasonic, the diameter of NFC decreased to $23.18 \mathrm{~nm}$ on average and the uniformity of NFC was improved. Approximately $40 \%$ of the NFC with diameter less than $20 \mathrm{~nm}$, and the NFC with a diameter less than $30 \mathrm{~nm}$ accounted for about $90 \%$. Compared with grinding treatment and HPH treatment, ultrasonic treatment can better destroy the amorphous part of cellulose and weaken the hydrogen bonding between cellulose chains.

\section{X-ray Diffraction Analysis}

The XRD patterns of NFC treated by chemical pretreatment, GT, GT-HPH, and GHPH-U are shown in Fig. 2. The diffractograms displayed the same typical cellulose-I crystal structure with the broad peaks at $2 \theta=15^{\circ}$ corresponding to the (110) crystallographic plane, and a sharp peak at $22.6^{\circ}$ corresponding to the (200) crystallographic plane.

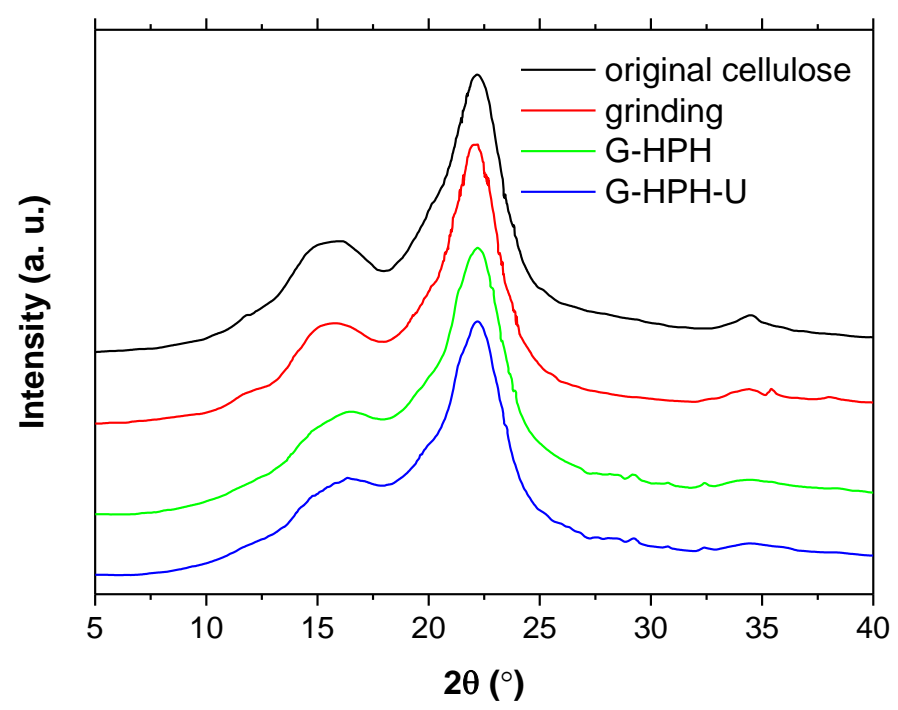

Fig. 2. X-ray diffraction pattern of (a) chemical pretreated cellulose, (b) GT treated NFC, (c) GT$\mathrm{HPH}$ treated NFC, and (d) GT-HPH-U treated NFC 
In all cases, the cellulose-I crystal structure was maintained in the mechanically treated NFC. This result indicates that the treatments such as grinding, high pressure homogenization, and ultrasonic had no obvious influence on the crystal structure of natural cellulose. By contrast, the degree of crystallinity of NFC was affected greatly by the mechanical treatment. In the process of GT, GT-HPH, and GT-HPH-U treatment on cellulose, these forces caused cleavage of the crystallites and an increase in non-crystalline parts (Ahmadzadeh et al. 2018). The crystallinity indexes of GT-, GT-HPH-, and GT-HPHU- treated NFC were $64.2 \%, 61.7 \%$, and $60.8 \%$, respectively, which is much higher than about $45 \%$ of original cellulose and lower than $65 \%$ of chemical pretreated cellulose. This indicates that the breakage of the intermolecular hydrogen bonds in NFC caused the collapse of crystal structure and the emergence of amorphous region during mechanical treatment.

\section{Thermostability Analysis}

The effect of structural properties on the thermal degradation of cellulose can be evaluated using the thermogravimetry technique; because the different polymers with various structures behave differently when undergo thermal degradation (Ahmadzadeh et al. 2018). The TGA curves of chemical pretreated cellulose and NFC are shown in Fig. 3. As can be seen, the onset decomposition temperature of original cellulose was $210^{\circ} \mathrm{C}$. By contrast, the thermostability of NFC produced by grinding, GT-HPH, and GT-HPH-U treatments were increased. Due to the thermal decomposition of aromatic hydrocarbons in residual lignin, the chemical pretreated bagasse cellulose degraded obviously at the initial stage of heating. Rapid quality degradation occurred in the range of $250{ }^{\circ} \mathrm{C}$ to $380{ }^{\circ} \mathrm{C}$, and the weight loss rate reached $86 \%$ when heated to $600{ }^{\circ} \mathrm{C}$. Correspondingly, NFC produced by grinding, GT-HPH, and GT-HPH-U treatments exhibited the high thermal stability with the onset decomposition temperature of near $300{ }^{\circ} \mathrm{C}$. When heated to $600{ }^{\circ} \mathrm{C}$, the thermal weight loss rates of the samples produced by GT, GT-HPH, and GT-HPH-U treatments were $69.2 \%, 73.3 \%$, and $74.4 \%$, respectively. This indicated that the destruction of crystallization in NFC caused the decrease of thermal stability under the shear action of mechanical forces, which is not significant.

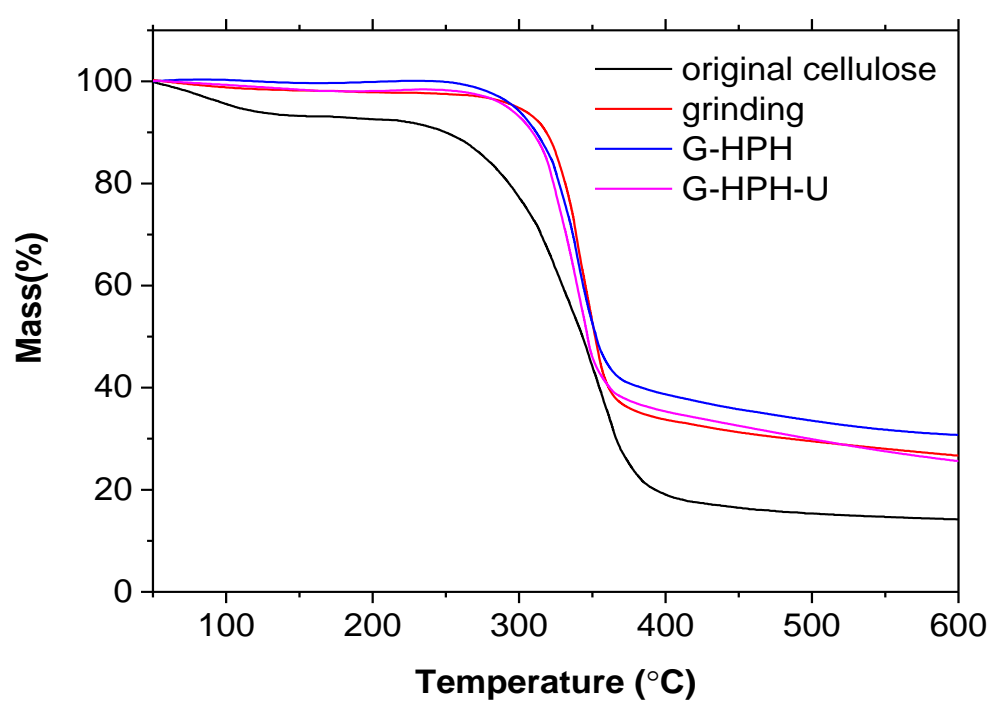

Fig. 3. TGA curves of (a) chemical pretreated cellulose, (b) GT treated NFC, (c) GT-HPH treated NFC, and (d) GT-HPH-U treated NFC 


\section{FTIR Analysis}

The FTIR characteristic peaks assigned to the functional groups identify the presence or removal of these components (Ahmadzadeh et al. 2018). FTIR spectra of chemically pretreated cellulose and NFC are shown in Fig. 4.

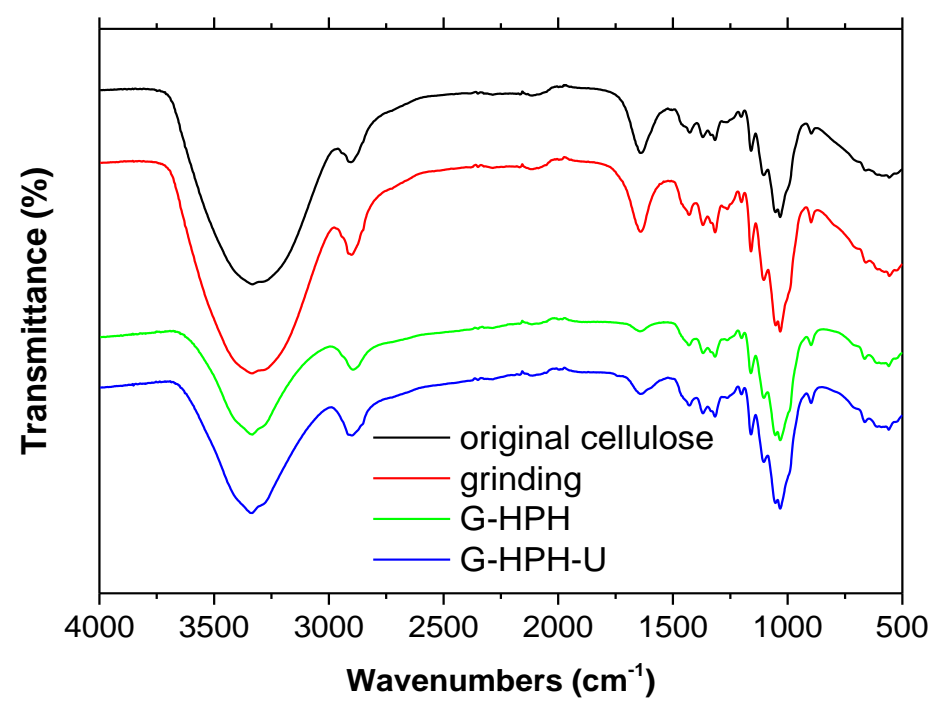

Fig. 4. IR spectra of (a) chemical pretreated cellulose, (b) GT treated NFC, (c) GT-HPH treated $\mathrm{NFC}$, and (d) GT-HPH-U treated NFC

The dominant peaks of $\mathrm{OH}$-stretching and $\mathrm{CH}$-stretching at approximately 3334 $\mathrm{cm}^{-1}$ and $2900 \mathrm{~cm}^{-1}$ were observed in the spectrum of all specimens. The absorbance band attributed to the stretching vibration of $\mathrm{C}=\mathrm{O}$ appeared at around $1640 \mathrm{~cm}^{-1}$. The shear vibration of $-\mathrm{CH}_{2}$ appeared as an absorption peak at $1420 \mathrm{~cm}^{-1}$. The absorption band at around $1023 \mathrm{~cm}^{-1}$ is related to the stretching vibration of $\mathrm{C}-\mathrm{O}$ in the alcohol hydroxyl groups of cellulose. The absorption peak near $895 \mathrm{~cm}^{-1}$ is related to the $\mathrm{C}-\mathrm{H}$ deformation vibration of cellulose. The above absorption peaks related to cellulose are all observed in the FTIR spectra of chemical pretreated cellulose and GT-, GT-HPH- and GT-HPH-Utreated NFC. Under the action of mechanical pretreatment, hemicellulose and lignin are almost removed, and highly purified NFC is produced (Ghaderi et al. 2014). Compared with the FTIR spectrum of chemical pretreated cellulose, the absorption peak located at $1731 \mathrm{~cm}^{-1}$ related to the stretching vibration of $\mathrm{C}=\mathrm{O}$ on the xylan acetyl group $\mathrm{CH}_{3} \mathrm{C}=\mathrm{O}$ in hemicellulose and the absorption peak at $1460 \mathrm{~cm}^{-1}$ related to the bending vibration of C$\mathrm{H}$ on the $-\mathrm{CH}_{2}$ of lignin disappeared in the FTIR spectra of NFC. Two absorption bands at $1640 \mathrm{~cm}^{-1}$ and $1508 \mathrm{~cm}^{-1}$ are related to the stretching vibration peak of carbonyl $\mathrm{C}=\mathrm{O}$ in the side chain of lignin and the vibrational absorption peak of benzene ring skeleton, respectively. These two bands were detected in the spectrum of chemical pretreated cellulose; however, their intensity was reduced in the spectra of NFC. There were no obvious differences in FTIR spectrum among NFC, which indicates that no other chemical reaction occurred during the processes of cellulose pretreated by grinding, high pressure homogenization, and ultrasonication. 


\section{Mechanical Property Analysis}

Figure 5 shows the effect of mechanical pretreatment on tensile strength of NFC. The mechanical performances of NFC films strongly depend on the size and morphology of their corresponding constituent NFC, which can be controlled by the mechanical treatments (Liu et al. 2020).

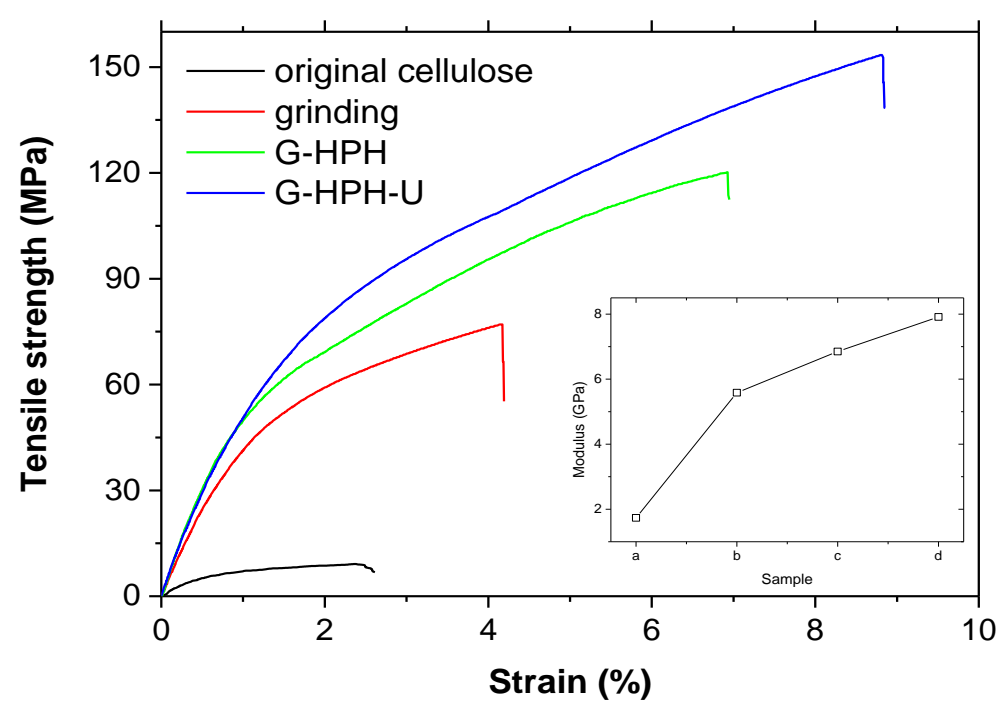

Fig. 5. Stress-strain curves and modulus curve of (a) chemical pretreated cellulose, (b) GT treated NFC, (c) GT-HPH treated NFC, and (d) GT-HPH-U treated NFC.

The tensile strength of NFC films increased with the increase of mechanical force. Compared with the chemical pretreated cellulose fiber, the tensile strength and the strain at break of NFC film treated by grinding increased by $765 \%$ and $64.2 \%$, and reached 77 $\mathrm{MPa}$ and $4.18 \%$, respectively. Under the shear action of HPH treatment, the aspect ratio and the size of NFC were further adjusted. Moreover, the tensile strength and strain at break of NFC treated by GT-HPH-U increased to $153.6 \mathrm{MPa}$ and $8.83 \%$, respectively. These results indicate that the strong mechanical force made the diameter distribution of NFC more uniform, the diameter smaller, and the specific surface area larger, which is verified by SEM in Fig. 1. Compared with the action of grinding process and HPH process, ultrasonic treatment can further individualized NFC (Nuruddin et al. 2011). A large number of hydroxyl groups on the NFC surface form a strong hydrogen bond between the adjacent fibers, which improves the interfacial bonding between the nanofibers (Ahmadzadeh et al. 2018). Under the action of HPH and ultrasonic treatment, the crystal region of NFC is partially destroyed, which is helpful to improve the elongation at break of NFC. The network structure formed by the interweaving of NFC and the uniformly distributed diameter reduce the stress concentration of NFC, which makes the NFC film have excellent mechanical properties. The prepared NFC can be used as reinforcement materials and matrix materials to provide good mechanical support for the matrix. 


\section{CONCLUSIONS}

1. Nanofibrillated cellulose (NFC) from sugarcane bagasse was prepared by grinding, high pressure homogenization, and ultrasonication coupled with simple chemical pretreatment. Compared with the complex chemical treatment, the grinding-high pressure homogenization-ultrasonic process is more convenient in the preparation of NFC, which resulted in NFC with the average diameter of $23.18 \mathrm{~nm}$ and higher thermal stability and crystallinity.

2. A very low-value agricultural waste product can be converted to a high performance NFC with tensile strength of $153.6 \mathrm{MPa}$ and strain at break of $8.83 \%$.

3. Under the action of mechanical force produced by GT-HPH-U, the multilayer structure of the cellulose was destroyed, and NFC with small diameter, uniform distribution, and large specific surface area was obtained. The strong hydrogen bonding interaction produced by a large number of hydroxyl groups on the surface of the nanofiber and the decrease of stress concentration caused by the uniformly dispersed fiber network structure greatly enhanced the mechanical properties of the NFC and improved the crystallinity and thermal stability of the fiber. By using the ultrasonic treatment, part of the cellulose crystal region was further destroyed, which increased the elongation at break while maintaining the high tensile strength of NFC, thus expanding the application range of NFC.

\section{ACKNOWLEDGMENTS}

The authors are grateful for the support of the Anhui Provincial Natural Science Foundation, Grant No. 1908085ME154; and the Open Fund of Fujian Universities and Colleges Engineering Research Center of Soft Plastic Packaging Technology for Food, Grant No. G1-KF1708.

\section{REFERENCES CITED}

Abraham, E., Deepa, B., Pothan, L. A., Jacob, M., Thomas, S., Cvelbar, U., and Anandjiwala, R. (2011). "Extraction of nanocellulose fibrils from lignocellulosic fibres: A novel approach," Carbohydrate Polymers 86(4), 1468-1475. DOI: 10.1016/j.carbpol.2011.06.034

Afra, E., Yousefi, H., Hadilam, M. M., and Nishino, T. (2013). "Comparative effect of mechanical beating and nanofibrillation of cellulose on paper properties made from bagasse and softwood pulps," Carbohydrate Polymers 97(2), 725-730. DOI: 10.1016/j.carbpol.2013.05.032

Ahmadzadeh, S., Nasirpour, A., Harchegani, M. B., Hamdami, N., and Keramat, J. (2018). "Effect of electrohydrodynamic technique as a complementary process for cellulose extraction from bagasse: Crystalline to amorphous transition," Carbohydrate Polymers 188, 188-196. DOI: 10.1016/j.carbpol.2018.01.109

Alila, S., Besbes, I., Vilar, M. R., Mutjé, P., and Boufi, S. (2013). "Non-woody plants as raw materials for production of microfibrillated cellulose (MFC): A comparative 
study," Industrial Crops and Products 41, 250-259. DOI:

10.1016/j.indcrop.2012.04.028

Bautista, E. G., Gutierrez, E., Dupuy, N., Gaime-Perraud, I., Ziarelli, F., and da Silva, A. M. F. (2019). "Pre-treatment of a sugarcane bagasse-based substrate prior to saccharification: Effect of coffee pulp and urea on laccase and cellulase activities of Pycnoporus sanguineus," Journal of Environmental Management 239, 178-186. DOI: 10.1016/j.jenvman.2019.03.033

Chen, P., Yu, H., Liu, Y., Chen, W., Wang, X., and Ouyang, M. (2013). "Concentration effects on the isolation and dynamic rheological behavior of cellulose nanofibers via ultrasonic processing," Cellulose 20(1), 149-157. DOI: 10.1007/s 10570-012-9829-7

de Moraes Rocha, G. J., Nascimento, V. M., Gonçalves, A. R., Silva, V. F. N., and Martín, C. (2015). "Influence of mixed sugarcane bagasse samples evaluated by elemental and physical-chemical composition," Industrial Crops and Products, 64, 52-58. DOI: 10.1016/j.indcrop.2014.11.003

Ghaderi, M., Mousavi, M., Yousefi, H., and Labbafi, M. (2014). “All-cellulose nanocomposite film made from bagasse cellulose nanofibers for food packaging application," Carbohydrate Polymers 104, 59-65. DOI: 10.1016/j.carbpol.2014.01.013

Gil-López, D. L., Lois-Correa, J. A., Sánchez-Pardo, M. E., Domínguez-Crespo, M. A., Torres-Huerta, A. M., Rodríguez-Salazar, A. E., and Orta-Guzmán, V. N. (2019). "Production of dietary fibers from sugarcane bagasse and sugarcane tops using microwave-assisted alkaline treatments," Industrial Crops and Products 135, 159169. DOI: 10.1016/j.indcrop.2019.04.042

Huq, T., Salmieri, S., Khan, A., Khan, R. A., Le Tien, C., Riedl, B., Fraschini, C., Bouchard, J., Uribe-Calderon, J., Kamal, M.R., and Lacroix, M. (2012). "Nanocrystalline cellulose (NCC) reinforced alginate based biodegradable nanocomposite film," Carbohydrate Polymers 90(4), 1757-1763. DOI: 10.1016/j.carbpol.2012.07.065

Jiang, Y., Liu, X., Yang, Q., Song, X., Qin, C., Wang, S., and Li, K. (2019). “Effects of residual lignin on composition, structure and properties of mechanically defibrillated cellulose fibrils and films," Cellulose 26(3), 1577-1593. DOI: 10.1007/s10570-01802229-4

Ju, Y. H., Huynh, L. H., Kasim, N. S., Guo, T. J., Wang, J. H., and Fazary, A. E. (2011). "Analysis of soluble and insoluble fractions of alkali and subcritical water treated sugarcane bagasse," Carbohydrate Polymers 83(2), 591-599. DOI: 10.1016/j.carbpol.2010.08.022

Khonngam, T., and Salakkam, A. (2019). "Bioconversion of sugarcane bagasse and dry spent yeast to ethanol through a sequential process consisting of solid-state fermentation, hydrolysis, and submerged fermentation," Biochemical Engineering Journal 150, 107284. DOI: 10.1016/j.bej.2019.107284

Li, J., Wei, X., Wang, Q., Chen, J., Chang, G., Kong, L., Su, J., and Liu Y. (2012). "Homogeneous isolation of nanocellulose from sugarcane bagasse by high pressure homogenization," Carbohydrate Polymers 90(4), 1609-1613. DOI:

10.1016/j.carbpol.2012.07.038

Liu, C. F., Sun, R. C., Zhang, A. P., and Ren, J. L. (2007). "Preparation of sugarcane bagasse cellulosic phthalate using an ionic liquid as reaction medium," Carbohydrate Polymers 68(1), 17-25. DOI: 10.1016/j.carbpol.2006.07.002

Liu, X., Jiang, Y., Wang, L., Song, X., Qin, C., and Wang, S. (2020). “Tuning of size and 
properties of cellulose nanofibers isolated from sugarcane bagasse by endoglucanaseassisted mechanical grinding," Industrial Crops and Products 146, 112201. DOI: 0.1016/j.indcrop.2020.112201

Macías-Almazán, A., Lois-Correa, J. A., Domínguez-Crespo, M. A., López-Oyama, A. B., Torres-Huerta, A. M., Brachetti-Sibaja, S. B., and Rodríguez-Salazar, A. E. (2020). "Influence of operating conditions on proton conductivity of nanocellulose films using two agroindustrial wastes: Sugarcane bagasse and pinewood sawdust," Carbohydrate Polymers 238, 116171. DOI: 10.1016/j.carbpol.2020.116171

Mazalevska, O., Struszczyk, M. H., Chrzanowski, M., and Krucińska, I. (2011).

"Application of electrospinning for vascular graft performance - Preliminary results," Fibres \& Textiles in Eastern Europe 19(4), 87. DOI: 10.1177/0040517511399966

Ninomiya, K., Abe, M., Tsukegi, T., Kuroda, K., Tsuge, Y., Ogino, C., Taki, K., Taima, T., Saito, J., Kimizu, M., Uzawa, K., and Takahashi, K. (2018). "Lignocellulose nanofibers prepared by ionic liquid pretreatment and subsequent mechanical nanofibrillation of bagasse powder: Application to esterified bagasse/polypropylene composites," Carbohydrate Polymers 182, 8-14. DOI: 10.1016/j.carbpol.2017.11.003

Nuruddin, M., Chowdhury, A., Haque, S. A., Rahman, M., Farhad, S., Jahan, M. S., and Quaiyyum, A. (2011). "Extraction and characterization of cellulose microfibrils from agricultural wastes in an integrated biorefinery initiative," Biomaterials, 3, 5-6. DOI: 10.1016/j.biombioe.2012.06.039

Rossignolo, J. A., Borrachero, M. V., Soriano, L., and Paya, J. (2018). "Influence of microwave oven calcination on the pozzolanicity of sugar cane bagasse ashes (SCBA) from the cogeneration industry," Construction and Building Materials 187, 892-902.

Segal, L. G. J. M. A., Creely, J. J., Martin Jr, A. E., and Conrad, C. M. (1959). “An empirical method for estimating the degree of crystallinity of native cellulose using the X-ray diffractometer," Textile Research Journal 29(10), 786-794. DOI: 10.1177/004051755902901003

Sun, X., Wu, Q., Zhang, X., Ren, S., Lei, T., Li, W., Xu G., and Zhang, Q. (2018). "Nanocellulose films with combined cellulose nanofibers and nanocrystals: tailored thermal, optical and mechanical properties," Cellulose 25(2), 1103-1115. DOI: 10.1007/s10570-017-1627-9

Tang, D., Noordover, B. A., Sablong, R. J., and Koning, C. E. (2012). “Thermoplastic poly(urethane urea)s from novel, bio-based amorphous polyester diols," Macromolecular Chemistry and Physics 213(23), 2541-2549. DOI: 10.1002/macp.201200397

Wang, Y., Wei, X., Li, J., Wang, F., Wang, Q., Chen, J., and Kong, L. (2015). "Study on nanocellulose by high pressure homogenization in homogeneous isolation," Fibers and Polymers 16(3), 572-578. DOI: 10.1007/s12221-015-0572-1

Article submitted: April 25, 2020; Peer review completed: June 4, 2020; Revised version received and accepted: July 4, 2020; Published: July 9, 2020.

DOI: 10.15376/biores. 15.3.6636-6647 